\title{
Optimal Routing for Multi-Hop Social-Based D2D Communications in the Internet of Things
}

\author{
Gaojie Chen, Member, IEEE, Jinchuan Tang, Student Member, IEEE, and Justin P. Coon, Senior Member, IEEE
}

\begin{abstract}
With the development of wireless communications and the intellectualization of machines, the Internet of things (IoT) has been of interest to both industry and academia. Multihop routing and relaying are key technologies that will underpin IoT mesh networks in the future. This paper investigates optimal routing based on the trusted connectivity probability (T-CP) for multi-hop, underlay, device-to-device (D2D) communications with decode-and-forward (DF) relaying. Both random and fixed locations for base stations (BSs) are considered, where the former case assumes that the locations of the BSs are modeled as a Poisson point process (PPP). First, we derive two expressions for the connectivity probability (CP): a tight lower bound and an exact closed-form. Analysis is carried out for the cases where the channel state information (CSI) between BSs and the D2D transmitter is known (CSI-aware) and unknown (no-CSI). Interference from active cellular users (CUEs) is characterized by modeling CUE locations as a PPP. Moreover, motivated by results that have shown that social behavior leads to D2D devices communicating with nearby neighbours, we derive the trust probability (TP) for D2D connections by using a rank-based model. Finally, we propose a novel routing algorithm that can achieve the highest T-CP for any pair of D2D devices in a distributed manner. The derived analytical results are verified by Monte Carlo simulations. We show that the proposed routing algorithm achieves almost the same performance as that attained through an exhaustive search. When BSs are located randomly, the optimal path based on the $\mathrm{CP}$ is the shortest path between the D2D transmitter and receiver. However, for fixed BSs, the optimal path selection depends on the locations of the BSs, which provides a very useful insight in designing the multi-hop D2D system for 5G IoT.
\end{abstract}

Index Terms-D2D communications, stochastic geometry, IoT, social behavior, routing.

\section{INTRODUCTION}

\section{A. Background Knowledge}

The emerging requirements of network ubiquity and machine intelligence to support and enhance future economic and social development have led to the Internet of Things (IoT) vision and have accelerated the research of technology aimed at achieving this vision [1]. Furthermore, the IoT promises to improve human lives by providing innovative services conceived for a wide range of application domains, ranging

This work was supported by EPSRC grants number EP/N002350/1 ("Spatially Embedded Networks") and EP/R006377/1 ("M3NETs").

J. Tang, and J. P. Coon are with the Department of Engineering Science, University of Oxford, Parks Road, Oxford, UK, OX1 3PJ, U.K., Emails: \{jinchuan.tang and justin.coon\}eeng.ox.ac.uk.

G. Chen is with the Department of Engineering, University of Leicester, Leicester, LE1 7RH, U.K., Email: gaojie.chen@leicester.ac.uk.

Copyright (c) 2012 IEEE. Personal use of this material is permitted. However, permission to use this material for any other purposes must be obtained from the IEEE by sending a request to pubs-permissions@ieee.org. from personal to industrial environments, and facing several societal challenges in various everyday-life human contexts [2]. A large number of heterogeneous and pervasive IoT devices continuously generating sensing data and connecting different technological areas, provides great opportunities for applications and services in the 5G. In order to achieve the purpose of IoT, Long Term Evolution-Advanced (LTE-A) is considered to play a fundamental role in the IoT arena providing a large coherent infrastructure and a wide wireless connectivity to the devices. However, LTE-A was originally designed to support high data rates and large data size, novel solutions are required to enable an efficient use of radio resources to convey small data packets typically exchanged by IoT applications in the large scale networks. Furthermore, the typically high energy consumption required by LTE-A is a serious obstacle to large-scale IoT deployments under cellular connectivity [1].

Device-to-device (D2D) communication has attracted a considerable amount of attention and is now regarded as a promising technology for IoT networks due to its high power efficiency, high spectral efficiency, and low transmission delay [3]-[6]. Such a flexible transmission protocol alleviates the stringent design requirements usually placed on infrastructure devices and reduces transmission overheads caused by centralized coordination and management [4]. D2D communications can be classified into two categories, depending on whether frequency resources are shared between D2D systems and traditional cellular systems; these two categories are termed underlay and overlay D2D communications, respectively [5]. It has been proved that underlay D2D communications would be able to provide high spectrum efficiency and support spectrum sharing in the 5G IoT [6].

Furthermore, a large number of devices in the IoT are based on human behavior [7], therefore, the social relationships (e.g., friendship and conflict) of people are very critical, which should be considered in the IoT with D2D communications. However, the systems in [4]-[6], [8]-[16] mostly assume that nodes are grouped into transmitter/receiver pairs. The nodes in each pair trust to each other by default, which is impractical. To overcome this problem, distance-based and rank-based trust models, which were proposed by [17] and [18], respectively, could be used to predict the trust probability (TP) for pair of nodes. By applying these models in uniformly distributed networks, the capacity of a wireless network was studied in [19] in the context of social groups. The rank-based model states that the TP depends on both the geographic distance and the node density [18] and [20], which is more accurate than the distance-based model. In this paper, therefore, we 
apply the rank-based trust model in multi-hop D2D networks to derive a routing algorithm aimed at maximizing the trusted connectivity probability (T-CP), which is a very comprehensive model to select optimal path for multi-hop social-based D2D communications in the $5 \mathrm{G}$ IoT.

\section{B. Related Works}

In order to enhance the capacity of cellular and D2D systems, an interference power control scheme was proposed in [8]. Furthermore, Yu et al. [9] investigated resource allocation and power control between cellular and D2D users and proposed an optimization method to improve system capacity. In [10], a full-duplex (FD) D2D aided cooperative nonorthogonal multiple access scheme aimed at enhancing outage performance was studied. Zheng et al. [11] proposed a scheme that allows D2D communications to underlay a cellular network through FD relaying. In [12], an outage performance analysis of FD relay-assisted D2D systems in a cellular network was provided.

The contributions summarized above only consider a small number of nodes. This is somewhat impractical considering the trend toward very dense networks, i.e. IoT. To address this issue, Hossain et al. utilized stochastic geometry, which has been provided in [13], to propose a practical and tractable analytical framework for D2D networks employing mode selection in [14]. Moreover, energy harvesting-based D2D communication in wireless networks was addressed in [15]. The majority of existing analysis only focuses on a single-hop D2D scenario. In [16], the outage probability for multi-hop D2D communications with shortest path routing was analyzed, although transmit power constraints of D2D devices were neglected in that work, which is an impractical assumption. $\mathrm{Xu}$ et al. in [21] considered a cross-layer multi-dimension optimization involving frequency, space, and time, to minimize the network average delay in the IoT. However, to consider the social behavior with optimal path selection for multi-hop D2D communications in $5 \mathrm{G}$ IoT is still an open question, which needs to be addressed.

In this paper, therefore, we apply the rank-based trust model in multi-hop, underlay, D2D networks in the IoT to derive a routing algorithm aimed at maximizing the trusted connectivity probability (T-CP). For the connectivity aspect of the work, we conduct a comprehensive connectivity probability (CP) analysis for the case where knowledge of channel state information (CSI) is available (CSI-aware) and unavailable (no-CSI). Our proposed routing algorithm can be easily implemented for the 5G IoT. The contributions of the paper are as follows:

- We obtain exact expressions and a lower bound for the CP for any two underlay D2D devices for random and fixed base station (BS) location models in the presence of interference arising from active cellular user equipment (CUEs).

- We firstly adopt the rank-based trust model to describe the impact of social relationships on the T-CP and propose a distributed routing algorithm to find the path from the source to the destination that maximizes the T-CP for multi-hop D2D communications in the IoT.
Table I

Notation AND Symbols USED IN THE PAPER

\begin{tabular}{|c|c|}
\hline Symbol & Definition/Explanation \\
\hline AWGN & additive white Gaussian noise \\
\hline BSs & base stations \\
\hline $\mathrm{CP}$ & connectivity probability \\
\hline CSI & channel state information \\
\hline CUEs & cellular users \\
\hline D2D & device-to-device \\
\hline DF & decode-and-forward \\
\hline FD & full-duplex \\
\hline HD & half-duplex \\
\hline IoT & Internet of things \\
\hline LTE-A & Long Term Evolution-Advanced \\
\hline PPP & Poisson point processs \\
\hline $\mathrm{T}-\mathrm{CP}$ & trusted connectivity probability \\
\hline$\rho_{B}$ and $\rho_{C}$ & density of BS and cellular user \\
\hline$N$ & the number of D2D relay \\
\hline$\alpha$ & path loss exponent \\
\hline $\mathbb{E}[\cdot]$ & expectation operation \\
\hline $\max _{k \in\{1 \ldots K\}}\left(x_{k}\right)$ & maximum function with a set \\
\hline $\min \left(x_{k}\right)$ & minimum function with a set \\
\hline$|\cdot|$ & the number of elements in the set \\
\hline$\|\cdot\|$ & distance operation \\
\hline $\mathcal{O}(x)$ & big $\mathrm{O}$ notation \\
\hline $\mathbb{P}(\cdot)$ & probability operator \\
\hline$R_{t h}$ & target rate \\
\hline$\Gamma(\cdot)$ & gamma function \\
\hline $\begin{array}{l}\mathcal{B}(\cdot) \\
\operatorname{erfc}(\cdot)\end{array}$ & $\begin{array}{l}\text { modified Bessel functions of the second kind } \\
\text { complementary error function }\end{array}$ \\
\hline
\end{tabular}

- We provide extensive simulations and numerical results to verify the theoretical analysis and illustrate the proposed routing algorithm. The results provide useful insight for designing practical systems according to different network parameters.

The remainder of the paper is organized as follows. Section II presents the system model. Section III analyzes the CP for any two D2D devices. Section IV proposes the secure routing algorithm to find the path with the highest T-CP for different scenarios. Section V gives numerical simulations to verify the analysis. Finally, section VI concludes the paper. The notation and symbols used in the paper are listed in Table I.

\section{System MODEL}

\section{A. Network Model}

The system model of the social-aware multi-hop D2D communications in the IoT is shown in Fig. 1, where the D2D transmitter $\left(D_{0}\right)$ transmits the information to the D2D destination $\left(D_{N+1}\right)$ by using the number of D2D DF relays $\left(U_{i}, i \in(1,2, \ldots, N)\right)$. All nodes are equipped with a single antenna and perform in the half-duplex mode. Without loss of generality, we locate the D2D transmitter and the D2D destination at the origin and a fixed location away from the origin, respectively, in a two-dimensional plane. Moreover, we assume that the locations of the cellular BSs and CUEs are modeled by using homogeneous Poisson point processs (PPP), $\Phi_{B}$ and $\Phi_{C}$, which have density $\rho_{B}$ and $\rho_{C}$, respectively. The noise variances are normalized to one, and the channels are quasi-static fading channels so that the channel coefficients 


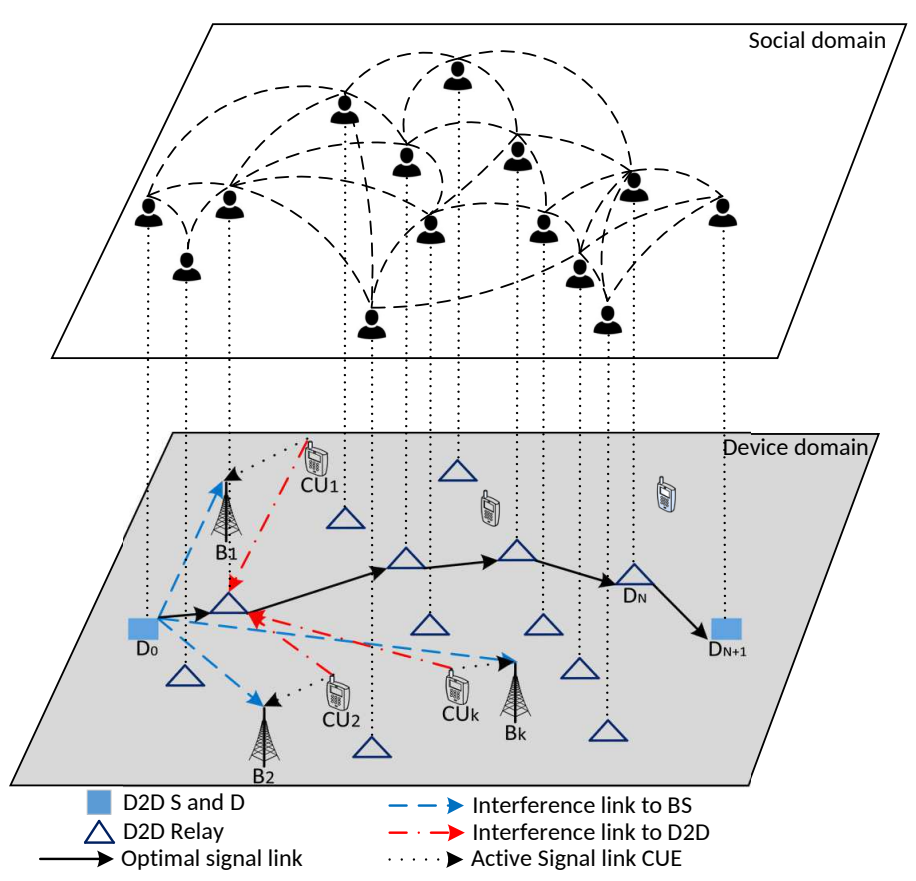

Figure 1. The proposed social-aware, multi-hop, underlay, D2D network architecture in the IoT.

remain unchanged during each transmission block duration but independently vary from one block time to another. In our work, all channels are assumed to undergo path loss and independent Rayleigh fading effects as $h_{i j}=\mu_{i j} d_{i j}^{-\alpha / 2}$, where $\alpha$ and $d_{i j}$ denote the pathloss exponent and the distance between two nodes, $i$ and $j$, respectively ${ }^{1}$. The fading coefficient $\mu_{i j}$ is a complex Gaussian random variable with unit variance. Therefore, the corresponding channel gains $\left|h_{i j}\right|^{2}$ are independently exponentially distributed with mean value $\lambda_{i j}$, and the average channel power is defined as $\lambda_{i j}=\mathbb{E}\left[\left|h_{i j}\right|^{2}\right]=d_{i j}^{-\alpha}$.

\section{B. Communication Model}

In this paper, we consider the underlay D2D scenario along with two cases that describe how much is known about the channels between BSs and D2D devices (CSI-aware and no-CSI). Hence, the transmit power for a D2D device is constrained by [12]

$$
P_{D}=\left\{\begin{array}{cl}
\min \left(\bar{P}_{D}, \frac{I_{t h}}{\max _{k \in \Phi_{B}}\left(\frac{\left|h_{i, k}\right|^{2}}{d_{i, k}^{\alpha}}\right)}\right), & \text { CSI-aware, } \\
\min \left(\bar{P}_{D}, \frac{I_{t h}}{\max _{k \in \Phi_{B}}\left(\frac{1}{d_{i, k}^{\alpha}}\right)}\right), & \text { no-CSI, }
\end{array}\right.
$$

where $\bar{P}_{D}$ is the maximum transmit power at the D2D user and $I_{t h}$ denotes a predefined interference power level at BSs. We suppose that the signals $x_{d}$ from node $D_{i}$ can be received

${ }^{1}$ We will typically consider the path loss exponent $\alpha=4$ for mathematical tractability and to provide insight into system performance. at the next node $D_{i+1}$. Therefore, the received signal at the $D_{i+1}$ can be written as:

$$
y_{i, i+1}=\sqrt{P_{D}} \frac{h_{i, i+1}}{d_{i, i+1}^{\alpha / 2}} x_{d}+\sum_{c \in \Phi_{C}}\left(\sqrt{P_{C}} \frac{h_{i, c}}{d_{i, c}^{\alpha / 2}}\right) x_{c}+n_{i+1},
$$

where $P_{C}$ denotes the transmit power of each CUE, and $n_{i+1}$ denotes the additive white Gaussian noise (AWGN) with variance $\sigma_{n}^{2}$ at nodes $D_{i+1}$. The second term of (2) is the interference from the active CUEs, since each CUE sends the signal $x_{c}$ to its closest BS [15]. Substituting (1) into (2), the instantaneous signal-to-interference ratio $(\mathrm{SIR})^{2}$ of $D_{i+1}$ is given as:

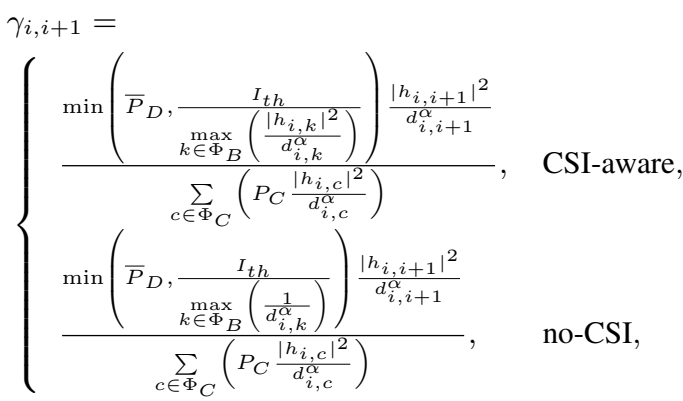

\section{Relationship Model}

In this paper, we considered the TP of D2D devices by using a rank-based trust model, which was proposed in [18] and [24] based on the analysis of daily experiences and real data traces from online social networks. Considering any two D2D devices $D_{i}$ and $D_{i+1}$, we define the rank of $D_{i+1}$ with respect to $D_{i}$ as

$$
\mathcal{R}_{i}(i+1)=\left|\left\{l:\left\|L_{l}-L_{i}\right\|<\left\|L_{i+1}-L_{i}\right\|\right\}\right|
$$

where $|\cdot|$ denotes the number of elements in the set, $\|\cdot\|$ denotes the distance operation, and $L_{x}$ denotes the location of $D_{x}$. Then the probability that $D_{i+1}$ is trusted by $D_{i}$ is

$$
\mathbb{P}_{i, i+1}^{(t)}=\frac{1}{G \mathcal{R}_{i}^{\beta}(i+1)},
$$

where $\beta$ is the parameter of the rank-based model and $G=\sum_{n=1}^{N} \frac{1}{n^{\beta}}$ is a normalizing factor. The rank-based model accounts for the majority of the friendships in the LiveJournal online community, as provided in [18]. Moreover, the work in [25] suggests that the model indeed guarantees smallworld properties, such that with geographical information only, a friendship chain with at most $\mathcal{O}\left(\log ^{3} n\right)$ hops can be established between an arbitrary source node and a target node chosen uniformly at random from the whole population, where $\mathcal{O}$ denotes big $\mathrm{O}$ notation.

\section{TRusted Connection Probability}

In this section, we investigate the T-CP between any two D2D devices based on the random and fixed BS location models. First, by using the mathematical definition of trusted

\footnotetext{
${ }^{2}$ The interference limited case is a standard approximation for the signalto-interference-plus-noise ratio (SINR) for systems operating in the high SNR region [22], [23].
} 
connection in [26] - specifically, two nodes can establish a trusted connection if they can communicate and trust each other - then the T-CP can be defined as:

$$
\mathbb{P}_{i, i+1}=\mathbb{P}_{i, i+1}^{(t)} \mathbb{P}_{i, i+1}^{(c)},
$$

where $\mathbb{P}_{i, i+1}^{(c)}$ denotes the CP between $D_{i}$ and $D_{i+1}$, which is given by:

$$
\mathbb{P}_{i, i+1}^{(c)}=\mathbb{P}\left(\log _{2}\left(1+\gamma_{i, i+1}\right)>R_{t h}\right),
$$

where $R_{t h}$ denotes the target rate.

\section{A. Random BS Locations}

From the system level perspective, the randomly located BS model is important since it provides average performance knowledge. Therefore, in this subsection, we will analyze the $\mathrm{CP}$ for this scenario based on the CSI-aware and no-CSI assumptions.

1) CSI-Aware: If the instantaneous CSI between BSs and D2D transmitters is known, the CP between $D_{i}$ and $D_{i+1}$ is given by

$$
\mathbb{P}_{i, i+1}^{(c)}=\mathbb{P}\left(\frac{\min \left(\bar{P}_{D}, \frac{I_{t h}}{\max _{k \in \Phi_{B}}\left(\frac{\left|h_{i, k}\right|^{2}}{d_{i, k}^{\alpha}}\right)}\right) \frac{\left|h_{i, i+1}\right|^{2}}{d_{i, i+1}^{\alpha}}}{\sum_{c \in \Phi_{C}}\left(P_{C} \frac{\left|h_{i, c}\right|^{2}}{d_{i, c}^{\alpha}}\right)}>\kappa\right)
$$

where $\kappa=2^{R_{t h}}-1$. Let $A=\max _{k \in \Phi_{B}}\left(\frac{\left|h_{i, k}\right|^{2}}{d_{i, k}^{\alpha}}\right)$ and

$$
B=\frac{\frac{\left|h_{i, i+1}\right|^{2}}{d_{i, i+1}^{\alpha}}}{\sum_{c \in \Phi_{C}}\left(P_{C} \frac{\left|h_{i, c}\right|^{2}}{d_{i, c}^{\alpha}}\right)} .
$$

Then the cumulative distribution function (CDF) of $A$ can be obtained as

$$
\begin{aligned}
F_{A}(x) & =\mathbb{P}\left(\max _{k \in \Phi_{B}}\left(\left|h_{i, k}\right|^{2}<x d_{i, k}^{\alpha}\right)\right) \\
& =\underset{k \in \Phi_{B}}{\mathbb{E}}\left[\prod_{k \in \Phi_{B}} e^{-x d_{i, k}^{\alpha}}\right] \\
& \stackrel{(a)}{=} \exp \left(-\rho_{B} \int_{0}^{2 \pi} \int_{0}^{\infty} r e^{-x r^{\alpha}} \mathrm{d} r \mathrm{~d} \theta\right) \\
& =\exp \left(-x^{-\frac{2}{\alpha} \Omega}\right)
\end{aligned}
$$

where (a) holds by using the probability generating functional lemma [13], $\Omega=\frac{2 \pi \rho_{B}}{\alpha} \Gamma\left(\frac{2}{\alpha}\right)$ and $\Gamma(\cdot)$ denotes the gamma function. Then the probability density function (PDF) of $A$ can be written as

$$
f_{A}(x)=x^{-\frac{2}{\alpha}-1} \frac{2 \Omega}{\alpha} \exp \left(-x^{-\frac{2}{\alpha} \Omega}\right) .
$$

Then the CDF and PDF of $B$ can be obtained as (12) at the top of the next page, where for brevity and ease of exposition, we let $t=\left|h_{i, i+1}\right|^{2}$ in (a) and the PDF of $t$ is $e^{-t}$, and (b) holds for the probability generating functional, and

$$
f_{B}(y)=\frac{2 \Psi}{\alpha} y^{\frac{2}{\alpha}-1} \exp \left(-y^{\frac{2}{\alpha}} \Psi\right)
$$

respectively, where $\Psi=\frac{\pi d_{i, i+1}^{2} \rho_{C} P_{C}^{\frac{2}{\alpha}}}{\operatorname{sinc}\left(\frac{2}{\alpha}\right)}$. Then we can obtain the lower bound on the CP between $D_{i}$ and $D_{i+1}$ by using the following lemma.

Lemma 1: The lower bound on the $\mathrm{CP}$ between $D_{i}$ and $D_{i+1}$ for the CSI-aware scenarios is given by

$$
\begin{aligned}
\mathbb{P}_{i, i+1}^{(c)}(\kappa) \geq & 2\left(\frac{\kappa}{I_{t h}}\right)^{\frac{1}{\alpha}} \sqrt{\Psi \Omega} \mathcal{B}\left(1,2\left(\frac{\kappa}{I_{t h}}\right)^{\frac{1}{\alpha}} \sqrt{\Psi \Omega}\right) \\
& -e^{\Omega\left(-\frac{I_{t h}}{\bar{P}_{D}}\right)^{-\frac{2}{\alpha}}}+e^{\Omega\left(-\frac{I_{t h}}{\bar{P}_{D}}\right)^{-\frac{2}{\alpha}}-\Psi\left(-\frac{\kappa}{\bar{P}_{D}}\right)^{\frac{2}{\alpha}}},
\end{aligned}
$$

where $\mathcal{B}(\cdot)$ denotes the modified Bessel functions of the second kind.

\section{Proof: See Appendix A.}

Remark 1: For given $d_{i, i+1}$, the CP between any two D2D devices for the CSI-aware case depends on the intensities of the BS and CUE processes, the path loss exponent $\alpha$, the transmit power of each CUE, and the threshold $I_{t h}$. Further analysis and the effect of these parameters on system performance are presented in section VI.

2) No-CSI: Suppose no information about the channels between the BSs and the D2D transmitters is available. This may be the case in, for example, super dense networks where there are not enough resources to perform accurate channel estimation. In this "no-CSI" case, the problem formulation depends only on distances between transmitters or, equivalently, the long-term average channel gains between BSs and D2D transmitters. Thus, the CP between $D_{i}$ and $D_{i+1}$ is given by

$$
\mathbb{P}_{i, i+1}^{(c)}=\mathbb{P}\left(\frac{\min \left(\bar{P}_{D}, \frac{I_{t h}}{\max _{k \in \Phi_{B}}\left(\frac{1}{d_{i, k}^{\alpha}}\right)}\right) \frac{\left|h_{i, i+1}\right|^{2}}{d_{i, i+1}^{\alpha}}}{\sum_{c \in \Phi_{C}}\left(P_{C} \frac{\left|h_{i, c}\right|^{2}}{d_{i, c}^{\alpha}}\right)}>\kappa\right) .
$$

Let $A_{1}=\max _{k \in \Phi_{B}}\left(\frac{1}{d_{i, k}^{\alpha}}\right)$. Then we have

$$
A_{1}=\max _{k \in \Phi_{B}}\left(\frac{1}{d_{i, k}^{\alpha}}\right)=\frac{1}{\left(\min _{k \in \Phi_{B}}\left(d_{i, k}\right)\right)^{\alpha}} .
$$

According to [27], the PDF of the distance $d_{i, *}=\min _{k \in \Phi_{B}}\left(d_{i, k}\right)$ for the nearest neighbor in a homogeneous PPP is given by

$$
f_{d_{i, *}}(x)=e^{-\rho_{B} \pi x^{2}} 2 \rho_{B} \pi x .
$$

Then the PDF of $A_{11}=d_{i, *}^{\alpha}$ can be obtained as

$$
f_{A_{11}}(x)=\frac{2 \rho_{B} \pi}{\alpha} x^{\frac{2}{\alpha}-1} e^{-x^{\frac{2}{\alpha}} \rho_{B} \pi} .
$$

According to the calculation of inverse distribution, we can obtain the PDF of $A_{1}=1 / A_{11}$ as

$$
f_{A_{1}}(x)=\frac{2 \rho_{B} \pi}{\alpha} x^{-\frac{2}{\alpha}-1} e^{-x^{-\frac{2}{\alpha}} \rho_{B} \pi} .
$$




$$
\begin{aligned}
& F_{B}(y)=\mathbb{P}\left(\frac{\frac{\left|h_{i, i+1}\right|^{2}}{d_{i, i+1}^{\alpha}}}{\sum_{c \in \Phi_{C}}\left(P_{C} \frac{\left|h_{i, c}\right|^{2}}{d_{i, c}^{\alpha}}\right)}<y\right)=1-\mathbb{E}\left[\prod_{c \in \Phi_{C}} e^{-y d_{i, i+1}^{\alpha} P_{C}\left|h_{i, i+1}\right|^{2} d_{i, c}^{-\alpha}}\right] \stackrel{(a)}{=} 1-\mathbb{E}_{\Phi_{C}}\left[\prod_{c \in \Phi_{C}} \int_{0}^{\infty} e^{-y d_{i, i+1}^{\alpha} P_{C} t d_{i, c}^{-\alpha}} e^{-t} \mathrm{~d} t\right] \\
& =1-\mathbb{E}_{\Phi_{C}}\left[\prod_{c \in \Phi_{C}} \frac{1}{1+P_{C} y\left(d_{i, i+1} / d_{i, c}\right)^{\alpha}}\right] \stackrel{(b)}{=} 1-\exp \left(\rho_{C} \int_{0}^{2 \pi} \int_{0}^{\infty}\left(\frac{-P_{C} y\left(d_{i, i+1} / r\right)^{\alpha}}{1+P_{C} y\left(d_{i, i+1} / r\right)^{\alpha}}\right) r \mathrm{~d} r \mathrm{~d} \theta\right)=1-\exp \left(-y^{\frac{2}{\alpha}} \Psi\right)
\end{aligned}
$$

We can obtain the CP between $D_{i}$ and $D_{i+1}$ without the knowledge of instantaneous CSI by

$$
\begin{aligned}
& \mathbb{P}_{i, i+1}^{(c)}= 1-\mathbb{P}\left(\min \left(\bar{P}_{D}, \frac{I_{t h}}{A_{1}}\right) B<\kappa\right) \\
&= 1-\int_{\frac{I_{t h}}{\bar{P}_{D}}}^{\infty} \int_{0}^{\frac{x \kappa}{I_{t h}}} f_{A_{1}}(x) f_{B}(y) \mathrm{d} y \mathrm{~d} x \\
&-\int_{0}^{\frac{I_{t h}}{\bar{P}_{D}}} \int_{0}^{\frac{\kappa}{\bar{P}_{D}}} f_{A_{1}}(x) f_{B}(y) \mathrm{d} y \mathrm{~d} x \\
&\left(\stackrel{a}{)} 2\left(\frac{\kappa}{I_{t h}}\right)^{\frac{1}{\alpha}} \sqrt{\Psi \rho_{B} \pi} \mathcal{B}\left(1,2\left(\frac{\kappa}{I_{t h}}\right)^{\frac{1}{\alpha}} \sqrt{\Psi \rho_{B} \pi}\right)\right. \\
& \quad-e^{\rho_{B} \pi\left(-\frac{I_{t h}}{\bar{P}_{D}}\right)^{-\frac{2}{\alpha}}}\left(1-e^{-\Psi\left(-\frac{\kappa}{P_{D}}\right)^{\frac{2}{\alpha}}}\right),
\end{aligned}
$$

where (a) holds by using Lemma 1.

Remark 2: For given $d_{i, i+1}$, the CP between any two D2D devices for the no-CSI case depends on the BS and CUE intensities, the path loss exponent $\alpha$, the transmit power of each CUE, and the threshold $I_{t h}$. Further analysis of the effects of these parameters on system performance are presented in section VI.

\section{B. Fixed BS Locations}

Here, we treat the case where BS locations are fixed and known.

1) CSI-Aware: If the instantaneous CSI between BSs and D2D transmitters is known, the CP between $D_{i}$ and $D_{i+1}$, with the $K$ fixed $\mathrm{BSs}$, is given by

$$
\mathbb{P}_{i, i+1}^{(c)}=\mathbb{P}\left(\frac{\min \left(\bar{P}_{D}, \frac{I_{t h}}{\max _{k \in\{1 \ldots K\}}\left(\frac{\left|h_{i, k}\right|^{2}}{d_{i, k}^{\alpha}}\right)}\right) \frac{\left|h_{i, i+1}\right|^{2}}{d_{i, i+1}^{\alpha}}}{\sum_{c \in \Phi_{C}}\left(P_{C} \frac{\left|h_{i, c}\right|^{2}}{d_{i, c}^{\alpha}}\right)}>\kappa\right) .
$$

Let $A_{2}=\max _{k \in\{1 \ldots K\}}\left(\frac{\left|h_{i, k}\right|^{2}}{d_{i, k}^{\alpha}}\right)$ and suppose all channels between BSs and D2D transmitters are independent but nonidentically distributed (i.n.i.d.). Then the CDF and PDF of $A_{2}$ can be written as

$$
\begin{gathered}
F_{A_{2}}(x)=\prod_{k=1}^{K}\left(1-e^{-d_{i, j}^{\alpha} x}\right), \\
f_{A_{2}}(x)=\sum_{k=1}^{K} d_{i, k}^{\alpha} e^{-d_{i, k}^{\alpha} x} \prod_{j=1, j \neq k}^{K}\left(1-e^{-d_{i, k}^{\alpha} x}\right),
\end{gathered}
$$

respectively. We can obtain the CP between $D_{i}$ and $D_{i+1}$ for $\alpha=4$ by using (20) as

$$
\begin{aligned}
\mathbb{P}_{i, i+1}^{(c)} & =1-\mathbb{P}\left(\min \left(\bar{P}_{D}, \frac{I_{t h}}{A_{2}}\right) B<\kappa\right) \\
& =1-\Xi_{1}(\kappa)-\Xi_{2}(\kappa)
\end{aligned}
$$

where $\Xi_{1}(\kappa)$ and $\Xi_{2}(\kappa)$ can be obtained as (25) and (26) at the top of the the next page, where $\varrho_{1}=\sqrt{\frac{\Psi^{2} \kappa}{\bar{P}_{D}}}+\frac{I_{t h}}{\bar{P}_{D}}\left(d_{i, 1}^{4}+d_{i, 2}^{4}\right)$ and $\operatorname{erfc}(\cdot)$ denotes the complementary error function.

Remark 3: For given $d_{i, i+1}$, the CP between any two D2D devices for the CSI-aware case depends on the location and number of BSs, the intensity of the CUEs $\rho_{C}$, the path loss exponent $\alpha$, the transmit powers of CUEs, and the threshold $I_{t h}$. Further analysis of the effects that these parameters have on system performance is presented in section VI.

2) No-CSI: The CP between $D_{i}$ and $D_{i+1}$ without the knowledge of CSI is given by

$$
\mathbb{P}_{i, i+1}^{(c)}=\mathbb{P}\left(\frac{\min \left(\bar{P}_{D}, \frac{I_{t h}}{\max _{k \in\{1 \ldots K\}}\left(\frac{1}{d_{i, k}^{\alpha}}\right)}\right) \frac{\left|h_{i, i+1}\right|^{2}}{d_{i, i+1}^{\alpha}}}{\sum_{c \in \Phi_{C}}\left(P_{C} \frac{\left|h_{i, c}\right|^{2}}{d_{i, c}^{\alpha}}\right)}>\kappa\right) .
$$

Due to the location of BSs are known, we let $A_{4}=$ $\min \left(\bar{P}_{D}, \frac{I_{t h}}{\max _{k \in\{1 \ldots K\}}\left(\frac{1}{d_{i, k}^{\alpha}}\right)}\right)$, which is a constant. Then according to (12), we can obtain the CP between $D_{i}$ and $D_{i+1}$ without the knowledge of instantaneous CSI by

$$
\mathbb{P}_{i, i+1}^{(c)}=\exp \left(-\left(\frac{\kappa}{A_{4}}\right)^{\frac{2}{\alpha}} \frac{\pi d_{i, i+1}^{2} \rho_{C} P_{C}^{\frac{2}{\alpha}}}{\operatorname{sinc}\left(\frac{2}{\alpha}\right)}\right)
$$

Remark 4: For given $d_{i, i+1}$, the CP between any two D2D devices for the no-CSI case depends on the locations and numbers of BSs, intensity of the CUE process, the path loss exponent $\alpha$, the transmit powers of CUEs, and the threshold $I_{t h}$. More details about the effects of these parameters on system performance are presented in section VI.

\section{Trusted Connectivity Routing Algorithm}

In the above section, the exact and lower bound expressions of the T-CP for both the cases of random and fixed BS locations were been studied. Based on these results, in this section, we study the routing problem, which is related to the 


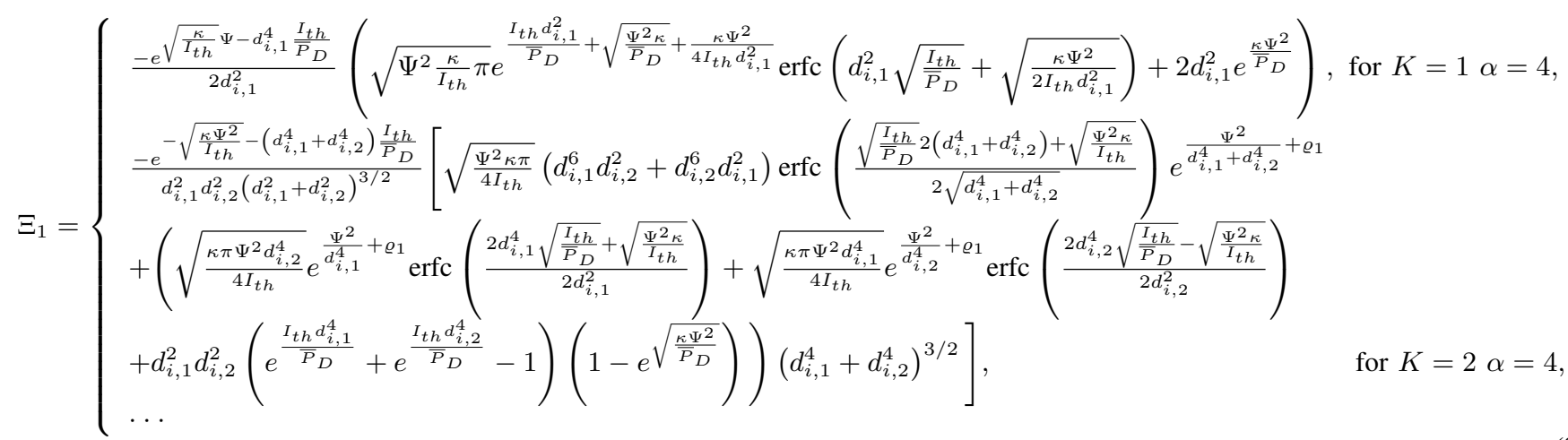

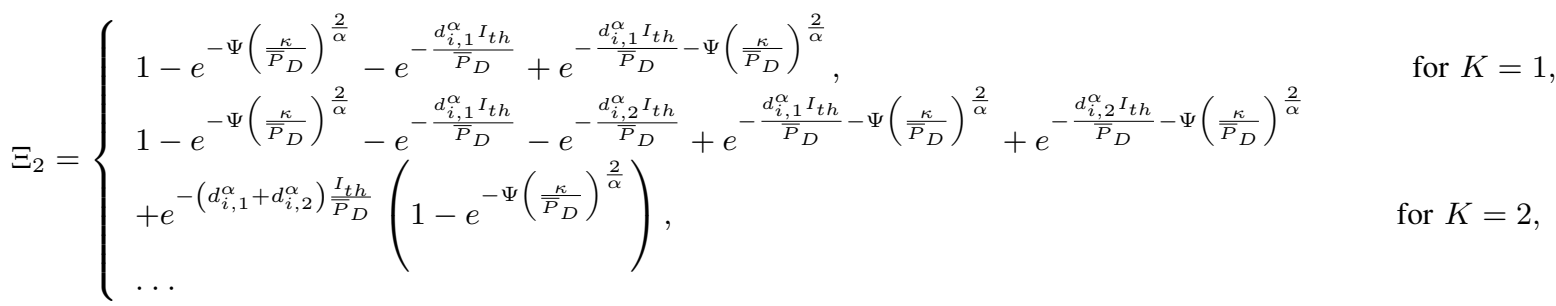

optimal path selection that achieves the maximum T-CP from the D2D transmitter to the receiver by using multiple D2D relays. Based on (6), the optimal path satisfies

$$
\mathbb{P}_{\mathrm{T}-\mathrm{CP}}\left(\Pi^{*}\right)=\max _{\Pi^{*} \in S_{\Pi}} \prod_{i \in S_{\Pi}} \mathbb{P}_{i, i+1}
$$

where $S_{\Pi}$ denotes the set of all potential paths between the D2D transmitter and receiver. It is obvious that we can solve this maximization problem by exhaustive search, but it would be computationally expensive. Motivated by the standard Dijkstra algorithm, we propose a new routing algorithm, which can return the maximum T-CP from a source vertex to all other vertices in a weighted graph.

Firstly, for the randomly located BS case, according to remarks 1 and 2, we can find the optimal path selection is independent of the BS locations and only dependent upon the locations of D2D devices. To the contrary, for the case of fixed BS locations, the optimal path selection will be influenced by the locations of BSs and D2D devices. Therefore, firstly, each D2D node needs to calculate the distances between itself and all other D2D devices and the BSs and store the topology information, which contains the neighbor list. Then by substituting the above analysis results (c.f., (5), (14), (20), (24) and (28)) with topological information into (6), we can obtain an adjacency T-CP matrix $\left(\mathbf{P} \in \mathcal{R}^{(N+2) \times(N+2)}\right)$. Finally, the optimal path with the maximum T-CP can be obtained by using the proposed routing algorithm summarized in Algorithm 1. At the beginning of processing, the network parameter initialization needs to be obtained. The first step is by using (5), (14), (20), (24) and (28) with the network parameters to obtain the adjacency T-CP matrix $\left(\mathbf{P} \in \mathcal{R}^{(N+2) \times(N+2)}\right)$ and then identifying the transmitter and destination node and setting transmitter as a permanent node and all other nodes to be temporary nodes. Then by using steps 4,5 and 6 to find the temporary node with maximum $\mathrm{T}-\mathrm{CP}$, update this temporary

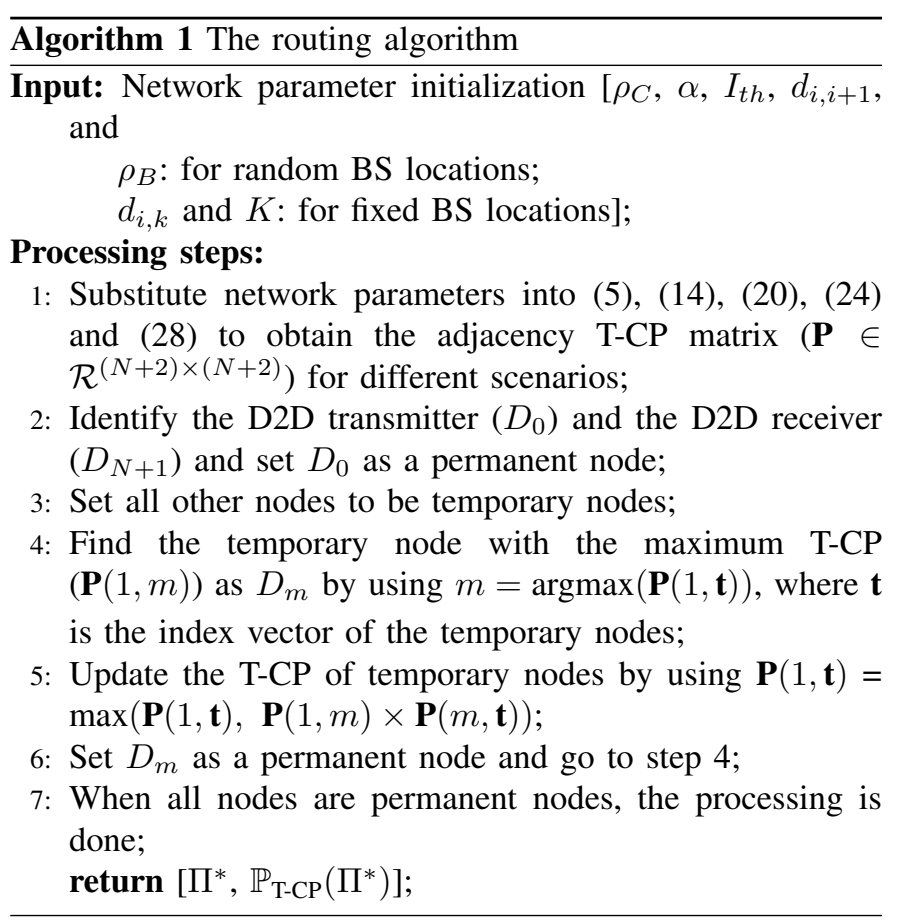

node and set it as a permanent node. By doing this iteration between steps 4, 5 and 6, when all notes are permanent nodes, we can obtain the optimal path and maximum T-CP of multihop D2D communication in the IoT.

The proposed routing algorithm gives a theoretical basis for selecting a path with the maximum T-CP. From Algorithm 1 , it is clear that the computational complexity is dominated by steps 2 to 7, which is similar to Dijkstra's algorithm. Hence, our proposed algorithm has the same level of computational complexity as the classical Dijkstra algorithm, which is $\mathcal{O}\left(N^{2}\right)$. It is polynomial and much lower than that of the 


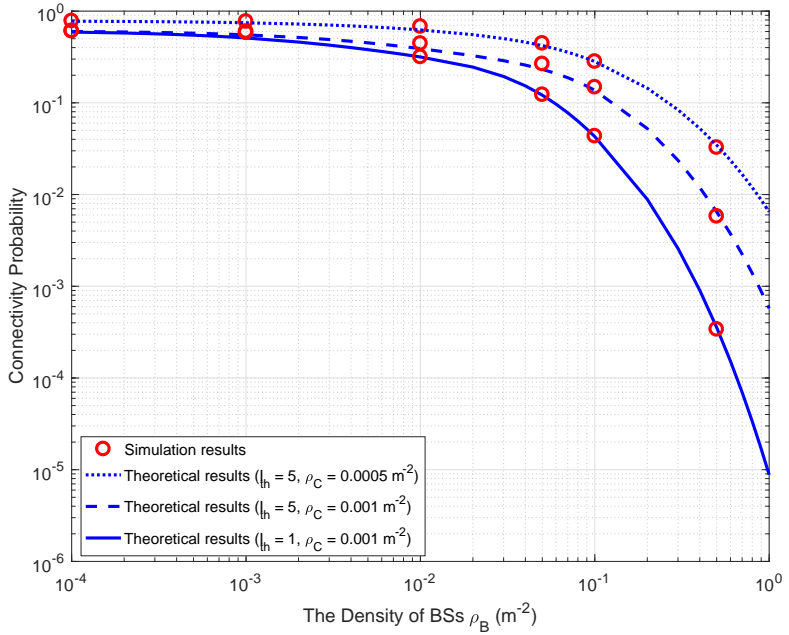

Figure 2. Theoretical vs. numerical CPs for a CSI-aware D2D pair in the presence of randomly located BSs.

exhaustive search, for which the complexity is $\mathcal{O}((N-2) !)$ [28]. In the next section, we give the comparison of optimal paths generated by our scheme and the exhaustive search.

\section{Simulations}

In this section, simulation results are given to verify the above analysis. Moreover, we assume the noise variance $\sigma_{n}^{2}=1$, and the simulation results are obtained by averaging over $10^{5}$ independent Monte Carlo (MC) trials. The path loss exponent is set to $\alpha=4$. We consider the exhaustive search based on MC simulation as a comparison benchmark.

\section{A. Connectivity Performance for a D2D Pair}

In this subsection, the $\mathrm{CP}$ of a D2D pair will be verified. Without loss of generality, the locations of the transmitter and receiver of a D2D pair are $(0,0)$ and $(0,10)$, respectively. For the case of randomly located BSs, we model the BSs as a homogeneous PPP $\Phi_{B}$ with density $\rho_{B}$. In Figs. 2 and 3, the comparison between theoretical (c.f. (14) and (20)) and numerical CP results for the CSI-aware and no-CSI cases are shown for different BS densities with $P_{D} / N_{0}=P_{C} / N_{0}=30 \mathrm{~dB}$. The simulation and theoretical results are shown to perfectly match. Moreover, it is clearly shown that the $\mathrm{CP}$ decreases with increasing BS density and/or decreasing peak interference power threshold $\left(I_{t h}\right)$. This follows due to a decrease in D2D transmit power. Furthermore, with increasing CUE density, the $\mathrm{CP}$ of the D2D pair decreases.

From the fixed topology perspective, we assume the locations of BSs are known to be $(5,5)$ and $(10,-15)$. Figs. 4 and 5 verify the $\mathrm{CP}$ for a D2D pair for the CSI-aware and no-CSI scenarios under different CUE densities and various power-to-noise ratios. The simulation and theoretical results are perfectly matched. Again, as with the case of randomly located BSs, it is clear from these results that the $\mathrm{CP}$ decreases as the density and power-to-noise ratio of each CUE node increases. To the contrary, with increasing D2D power-to-noise ratio, the $\mathrm{CP}$ of the $\mathrm{D} 2 \mathrm{D}$ increases.

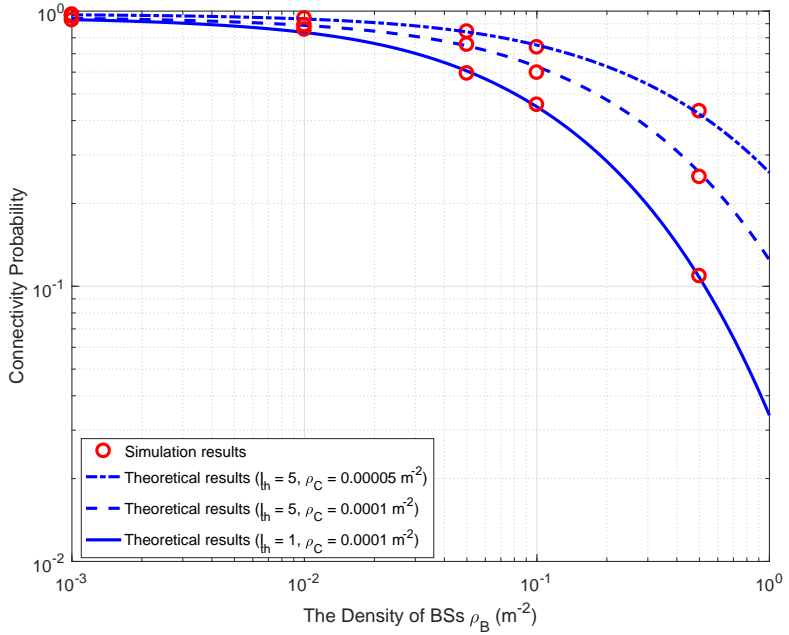

Figure 3. Theoretical vs. numerical CPs for a D2D pair with no-CSI in the presence of randomly located BSs.

\section{B. Performance of Optimal Routing Selection}

In this subsection, in order to give a clear comparison, we consider a multi-hop D2D network with a rank-based trust model where D2D relay nodes $\left(N_{R}=20\right)$ are randomly located on a $50 \times 50 \mathrm{~m}^{2}$ square area. The source and destination are located at $(-25,0)$ and $(25,0)$, respectively. We consider path selection based on both $\mathrm{CP}$ and T-CP calculation for the cases of randomly located and fixed BSs. Furthermore, for the parameter of the rank-based model, $\beta=1$ is assumed [18], [29]. Also, $\alpha=4, I_{t h}=5$, and $R_{t h}=1 \mathrm{bit} / \mathrm{s} / \mathrm{Hz}$.

In Fig. 6, we give the optimal path based on both the $\mathrm{CP}$ (c.f. (14)) and T-CP (c.f. (5) and (14)) metrics in a snapshot of the network for the case of randomly located BSs, where $\rho_{B}=0.0001 \mathrm{~m}^{-2}$. It is clear that the proposed exact route (T. R.) matches with the benchmark route that uses the exhaustive search (E. R.). We also compare the end-to-end CP and T-CP between the source and destination for the different densities of CUE in Table II. It is shown that the CP and T-CP of the proposed schemes are close to the benchmark. Moreover, with increasing CUE density, the CP and T-CP both decrease. According to (14), the CP only depends on the distance $d_{i, i+1}$; therefore, the optimal path for the CP metric is the shortest path between the transmitter and receiver. However, for T-CP, the trust probability has to be considered with the rank-based trust model; therefore, the optimal path for the T-CP metric is selected from the low density area for D2D relays, i.e., the area at the bottom of Fig. 6 . In other words, if two paths can guarantee a similar $\mathrm{CP}$, we are required to select a path in which the density of D2D relay devices is low to increase the probability of T-CP.

For the case of fixed BS locations, without loss of generality, we assume the locations of BSs are fixed at $(-10,-2)$ and $(-5$, 0 ), respectively. Fig. 7 shows the optimal path in a snapshot of the network based on $\mathrm{CP}$ and T-CP metrics. Again, the theoretically optimal path generated with the help of (25) is well matched to the simulation results obtained by an exhaustive search. It is clear that the optimal link for the $\mathrm{CP}$ metric changes when one BS is close to the center of the 


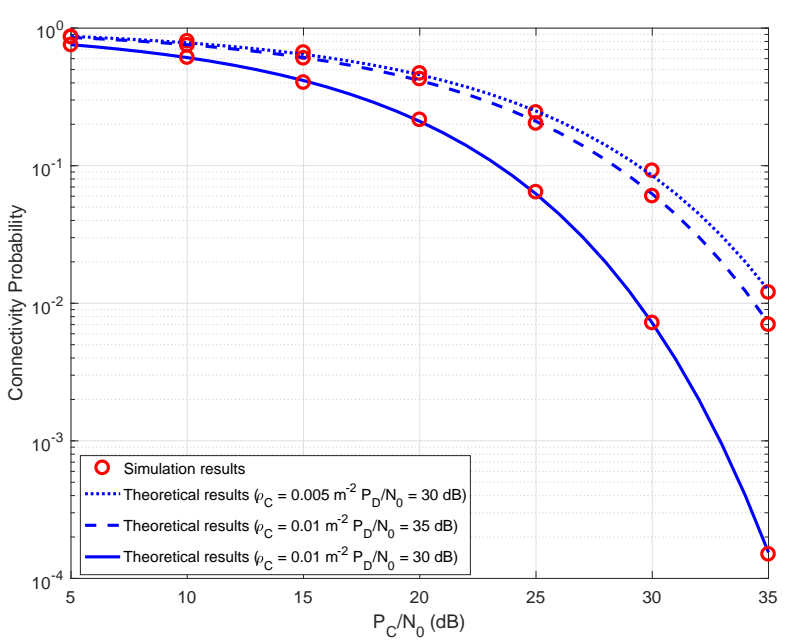

Figure 4. Theoretical vs. numerical CPs for a CSI-aware D2D pair with in the presence of fixed BSs.

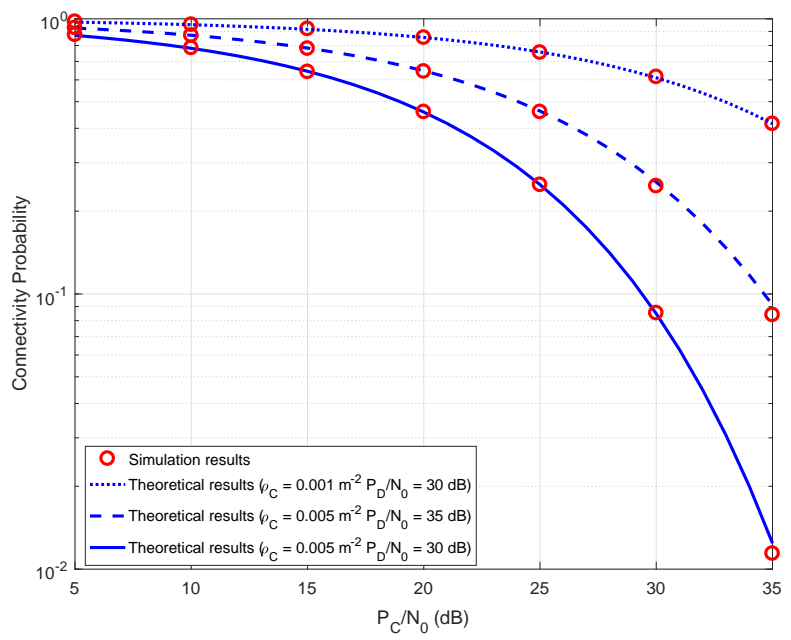

Figure 5. Theoretical vs. numerical CPs for a D2D pair no-CSI in the presence of fixed BSs.

Table II

THE COMPARISON OF END-TO-END CP AND T-CP WITH THE DIFFERENT CUE DENSITIES FOR THE CASE OF RANDOMLY LOCATED BSS.

\begin{tabular}{|c|c|c|c|}
\hline & $\rho_{C}=10^{-5}$ & $\rho_{C}=10^{-4}$ & $\rho_{C}=2 \times 10^{-4}$ \\
\hline E. R. (CP) & 0.9736 & 0.7778 & 0.5995 \\
\hline T. R. (CP) & 0.9744 & 0.7733 & 0.5984 \\
\hline E. R. (T-CP) & 0.0025 & 0.0019 & 0.0015 \\
\hline T. R. (T-CP) & 0.0025 & 0.0019 & 0.0015 \\
\hline
\end{tabular}

D2D relay path, because the distant D2D relay node will be selected to avoid interfering with the BS. By doing so, when we can estimate the locations of the BSs, we can design an optimal path to obtain the maximum CP. However, the social behavior may affect the T-CP in a similar manner to the case of randomly located BSs; therefore, the optimal path for T-CP is selected from the low density area. Again, the CP and T-CP decrease as the density of CUEs increases, as shown in Table III.

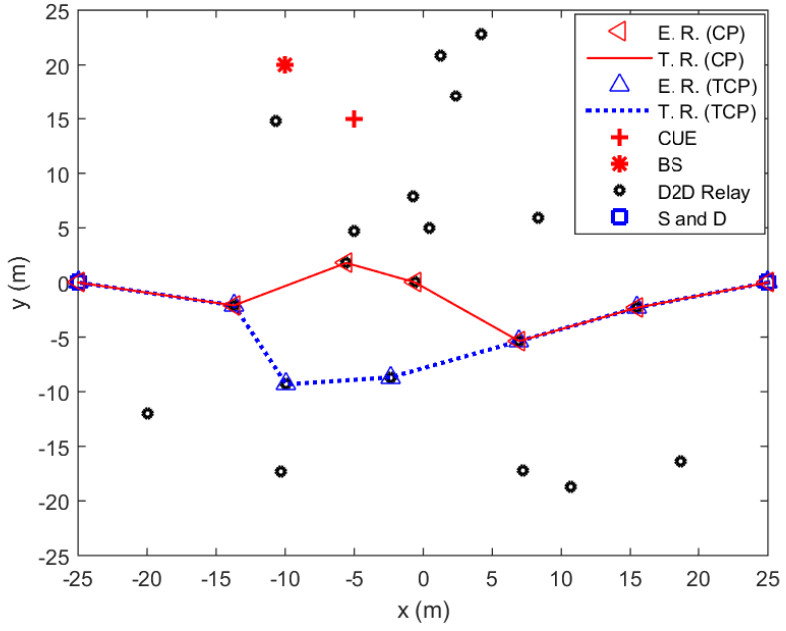

Figure 6. The optimal path in a snapshot of the network based on $\mathrm{CP}$ and T-CP metrics in the case of randomly located BSs, where $\alpha=4$, $I_{t h}=5, R_{t h}=1 \mathrm{bit} / \mathrm{s} / \mathrm{Hz}$, and $\rho_{B}=0.0001 \mathrm{~m}^{-2}$.

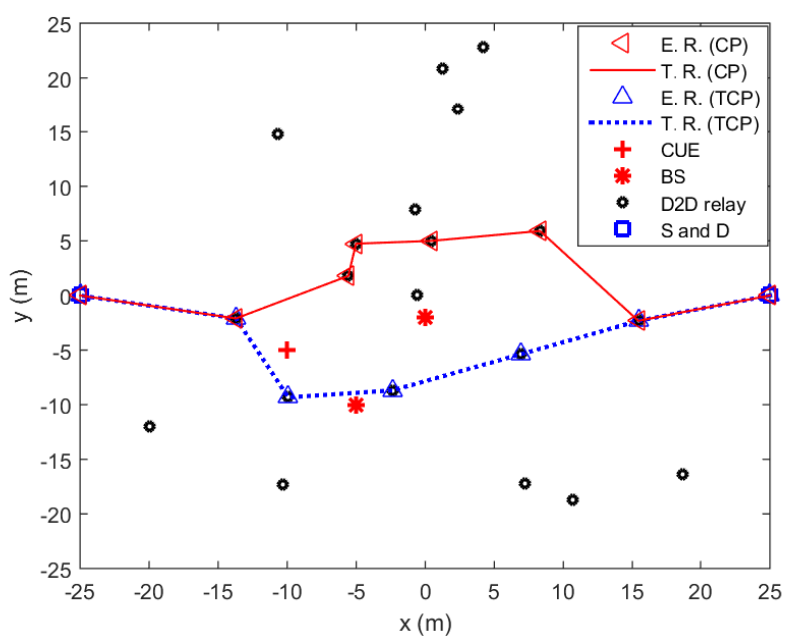

Figure 7. The optimal path in a snapshot of the network based on $\mathrm{CP}$ and T-CP metrics in the case of fixed BS locations, where $\alpha=4$, $I_{t h}=5$, and $R_{t h}=1 \mathrm{bit} / \mathrm{s} / \mathrm{Hz}$.

Table III

THE COMPARISON OF END-TO-END CP AND T-CP WITH THE DIFFERENT DENSITIES OF CUE FOR THE CASE OF FIXED LOCATED BS.

\begin{tabular}{|c|c|c|c|}
\hline & $\rho_{C}=10^{-5}$ & $\rho_{C}=10^{-4}$ & $\rho_{C}=2 \times 10^{-4}$ \\
\hline E. R. (CP) & 0.9740 & 0.7705 & 0.5935 \\
\hline T. R. (CP) & 0.9742 & 0.7698 & 0.5926 \\
\hline E. R. (T-CP) & 0.0025 & 0.0019 & 0.0014 \\
\hline T. R. (T-CP) & 0.0025 & 0.0019 & 0.0014 \\
\hline
\end{tabular}

\section{CONCLUSION}

In this paper, we studied the routing problem based on $\mathrm{CP}$ and T-CP metrics in wireless multi-hop underlay D2D communications with DF relaying. Both random and fixed locations of the BSs have been examined. The CPs for D2D pairs operating in the presence of CUE interference were characterized for the CSI-aware and no-CSI cases. Moveover, a rank-based trust model was provided to measure the TP between two D2D devices. Finally, we proposed a novel 
routing algorithm that can achieve the highest $\mathrm{T}-\mathrm{CP}$ for any pair of D2D devices in a distributed manner. The proposed routing algorithm achieves almost the same performance as given by an exhaustive search. According to simulations, for the case of randomly located BSs, the optimal path based on the CP metric is the shortest path between the D2D transmitter and receiver. However, for the case of fixed BS locations, the analysis showed that the optimal path depends on the locations of the BSs, which provides useful insight for designing multihop D2D systems for 5G IoT applications.

\section{APPENDIX A: PROOF OF Lemma 1}

According to (8) and (9), the $\mathrm{CP}$ is given by

$$
\begin{array}{r}
\mathbb{P}_{i, i+1}^{(c)}=1-\mathbb{P}\left(\min \left(\bar{P}_{D}, \frac{I_{t h}}{A}\right) B<\kappa\right) \\
\stackrel{(a)}{=} 1-\int_{\frac{I_{t h}}{\bar{P}_{D}}}^{\infty} \int_{0}^{\frac{x \kappa}{I_{t h}}} f_{x y}(x, y) \mathrm{d} y \mathrm{~d} x \\
\quad-\int_{0}^{\frac{I_{t h}}{\bar{P}_{D}}} \int_{0}^{\frac{\kappa}{\bar{P}_{D}}} f_{x y}(x, y) \mathrm{d} y \mathrm{~d} x, \\
\stackrel{(b)}{>} 1-\int_{0}^{\infty} \int_{0}^{\frac{x \kappa}{I_{t h}}} f_{x y}(x, y) \mathrm{d} y \mathrm{~d} x \\
-\int_{0}^{\frac{I_{t h}}{\bar{P}_{D}}} \int_{0}^{\frac{\kappa}{\bar{P}_{D}}} f_{x y}(x, y) \mathrm{d} y \mathrm{~d} x,
\end{array}
$$

where $f_{x y}(x, y)$ is a joint PDF, which can be calculated by using (11) and (13). The expression to the right of (a) cannot be provided in closed form. Hence, using [30], (14) can be obtained by using a lower bound (b), because the peak interference power is smaller than the transmit power of the D2D $\left(I_{t h} \ll \bar{P}_{D}\right)$, which can guarantee a prescribed quality of service for cellular communications. This concludes the proof.

\section{REFERENCES}

[1] C. T. Cheng, N. Ganganath, and K. Y. Fok, "Concurrent data collection trees for IOT applications," IEEE Transactions on Industrial Informatics, vol. 13, no. 2, pp. 793-799, April 2017.

[2] A. I. L. Atzori and G. Morabito, "The Internet of Things: A survey," Computer networks, vol. 54, no. 15, p. 27872805, 2010.

[3] O. Bello and S. Zeadally, "Intelligent device-to-device communication in the internet of things," IEEE Systems Journal, vol. 10, no. 3, pp. 11721182, Sep. 2016.

[4] A. Asadi, Q. Wang, and V. Mancuso, "A survey on device-to-device communication in cellular networks," IEEE Commun. Surveys Tutorials, vol. 16, no. 4, pp. 1801-1819, April 2014.

[5] M. Sheng, Y. Li, X. Wang, J. Li, and Y. Shi, "Energy efficiency and delay tradeoff in device-to-device communications underlaying cellular networks," IEEE J. Sel. Areas in Commun., vol. 34, no. 1, pp. 92-106, Jan. 2016.

[6] M. N. Tehrani, M. Uysal, and H. Yanikomeroglu, "Device-to-device communication in $5 \mathrm{G}$ cellular networks: challenges, solutions, and future directions," IEEE Commun. Mag., vol. 52, no. 5, pp. 86-92, May 2014.

[7] S. M. J. Gubbi, R. Buyya and M. Palaniswami, "Internet of things (iot): A vision, architectural elements, and future directions," Future Gener. Comput. Syst., vol. 29, no. 7, p. 16451660, Sep. 2013.

[8] H. Min, J. Lee, S. Park, and D. Hong, "Capacity enhancement using an interference limited area for device-to-device uplink underlaying cellular networks," IEEE Trans. Wireless Commun., vol. 10, pp. 3995-4000, Dec. 2011.

[9] C. H. Yu, K. Doppler, C. B. Ribeiro, and O. Tirkkonen, "Resource sharing optimization for device-to-device communication underlaying cellular networks," IEEE Trans. Wireless Commun., vol. 10, pp. 2752 2763, Aug. 2011.
[10] Z. Zhang, Z. Ma, M. Xiao, Z. Ding, and P. Fan, "Full-duplex device-todevice-aided cooperative nonorthogonal multiple access," IEEE Trans. Veh. Tech., vol. 66, no. 5, pp. 4467-4471, May 2017.

[11] G. Zhang, K. Yang, P. Liu, and J. Wei, "Power allocation for fullduplex relaying-based $\mathrm{d} 2 \mathrm{~d}$ communication underlaying cellular networks," IEEE Trans. Veh. Tech., vol. 64, no. 10, pp. 4911-4916, Oct. 2015.

[12] S. Dang, G. Chen, and J. P. Coon, "Outage performance analysis of full-duplex relay-assisted device-to-device systems in uplink cellular networks," IEEE Trans. Veh. Tech., vol. 66, pp. 4506-4510, May 2017.

[13] M. Haenggi, "Stochastic geometry for wireless networks," Cambridge Univ. Press, 2012.

[14] H. ElSawy, E. Hossain, and M. S. Alouini, "Analytical modeling of mode selection and power control for underlay D2D communication in cellular networks," IEEE Trans. Commun., vol. 62, pp. 4147-4161, Nov. 2014.

[15] A. H. Sakr and E. Hossain, "Cognitive and energy harvesting-based D2D communication in cellular networks: Stochastic geometry modeling and analysis," IEEE Trans. Commun., vol. 63, pp. 1867-1880, May 2015.

[16] S. Wang, W. Guo, Z. Zhou, Y. Wu, and X. Chu, "Outage probability for multi-hop D2D communications with shortest path routing," IEEE Commun. Lett., vol. 19, no. 11, pp. 1997-2000, Nov. 2015.

[17] J. M. Kleinberg, "Navigation in a small world," Nature, vol. 406, p. 845 , 2000.

[18] D. Liben-Nowell, J. Novak, R. Kumar, P. Raghavan, A. Tomkins, and R. L. Graham, "Geographic routing in social networks," Proc. Nat. Acad. Sci. United States Amer, vol. 102, no. 33, pp. 11623-11628, 2005.

[19] B. Azimdoost, H. R. Sadjadpour, and J. J. Garcia-Luna-Aceves, "Capacity of wireless networks with social behavior," IEEE Trans. Wireless Commun., vol. 12, no. 1, pp. 60-69, Jan. 2013.

[20] C. Wang, L. Shao, Z. Li, L. Yang, X. Y. Li, and C. Jiang, "Capacity scaling of wireless social networks," IEEE Trans. Parallel and Distrib. Syst., vol. 26, no. 7, pp. 1839-1850, July 2015.

[21] C. Xu, J. Feng, Z. Zhou, J. Wu, and C. Perera, "Cross-layer optimization for cooperative content distribution in multihop device-to-device networks," IEEE Internet of Things Journal, vol. PP, no. 99, pp. 1-1, 2017.

[22] G. Chen, J. P. Coon, and M. D. Renzo, "Secrecy outage analysis for downlink transmissions in the presence of randomly located eavesdroppers," IEEE Trans. Inf. Forensics and Security, vol. 12, pp. 1195-1206, May 2017.

[23] G. Chen, Y. Gong, P. Xiao, and J. A. Chambers, "Dual antenna selection in secure cognitive radio networks," IEEE Trans. Veh. Technol., vol. 65, no. 10, pp. 7993-8002, Oct. 2016.

[24] M. Cha, A. Mislove, and K. P. Gummadi, "A measurement-driven analysis of information propagation in the flickr social network," in Proc. ACM World Wide Web (WWW), New York, NY, USA, pp. 721730, March.

[25] R. Kumar, D. Liben-Nowell, J. Novak, P. Raghavan, and A. Tomkins, "Theoretical analysis of geographic routing in social networks," MIT Press, Cambridge, MA, USA, Tech. Rep. MIT-CSAIL-TR-2005-040, 2005.

[26] J. P. Coon, "Modelling trust in random wireless networks," in in Proc. IEEE ISWCS, Barcelona, Spain, pp. 976-981, Aug. 2014.

[27] M. Haenggi, "On distances in uniformly random networks," IEEE Trans. Inf. Theory, vol. 51, no. 10, pp. 3584-3586, Oct. 2005.

[28] C. E. Perkins and E. M. Royer, "Ad-hoc on-demand distance vector routing," in IEEE WMCSA Workshop, New Orleans, USA, Feb. 1999.

[29] C. Wang, H. M. Wang, X. G. Xia, and C. Liu, "Uncoordinated jammer selection for securing SIMOME wiretap channels: A stochastic geometry approach," IEEE Transactions on Wireless Communications, vol. 14, pp. 2596-2612, May 2015.

[30] I. S. Gradshteyn and I. M. Ryzhik, "Table of integrals, series, and products," Elsevier Academic Press, 7th ed. 2007. 


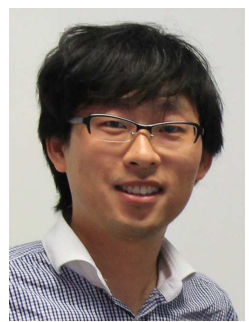

Gaojie Chen (S'09 - M'12) received the B.Eng. and B.Ec. degrees in electrical information engineering and international economics and trade from Northwest University, China, in 2006, and the M.Sc. (Hons.) and Ph.D. degrees in electrical and electronic engineering from Loughborough University, Loughborough, U.K., in 2008 and 2012, respectively. From 2008 to 2009, he was a Software Engineering with DTmobile, Beijing, China, and from 2012 to 2013, he was a Research Associate with the School of Electronic, Electrical and Systems Engineering, Loughborough University. He was a Research Fellow with 5GIC, Faculty of Engineering and Physical Sciences, University of Surrey, U.K., from 2014 to 2015. Then he was a Research Associate with the Department of Engineering Science, University of Oxford, U.K., from 2015 to 2018. He is currently a Lecturer with the Department of Engineering, University of Leicester, U.K. His current research interests include information theory, wireless communications, cooperative communications, cognitive radio, secrecy communication, and random geometric networks.

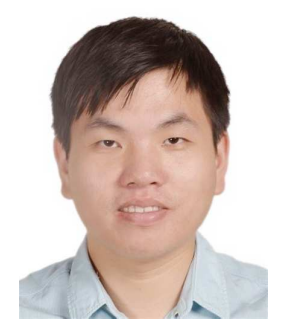

Jinchuan Tang ( $\left.S^{\prime} 17\right)$ received his B.Eng. degree in electronic information engineering from Chongqing University of Posts and Telecommunications in 2011. He worked as an Assistant Engineer in Huawei Technologies Co., Ltd. from 2011 to 2012. He obtained the M.Sc. degree with Distinction in wireless communications from the University of Southampton in early 2014 . He is currently pursuing a DPhil degree under the supervision of Professor Justin P. Coon with the Department of Engineering Science, University of Oxford. His current research interests include optimum power allocation, random geometric networks, secure communication, and route selection in wireless networks.

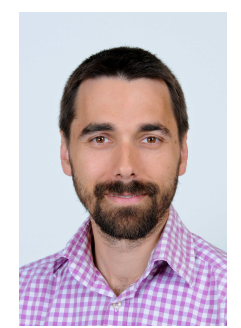

Justin P. Coon (S'02 - M'05 - SM'10) received the B.Sc. degree (Hons.) in electrical engineering from the Calhoun Honours College, Clemson University, USA, and the Ph.D. degree in communications from the University of Bristol, U.K., in 2000 and 2005, respectively. In 2004, he joined as a Research Engineer with the Bristol-based Telecommunications Research Laboratory (TRL), Toshiba Research Europe Ltd., where he was involved in research on a broad range of communication technologies and theories, including single- and multi-carrier modulation techniques, estimation and detection, diversity methods, and system performance analysis and networks. He held the research manager position from 2010 to 2013, during which time he led all theoretical and applied research on the physical layer at TRL. He was a Visiting Fellow with the School of Mathematics, University of Bristol, from 2010 to 2012, where he held a reader position with the Department of Electrical and Electronic Engineering from 2012 to 2013. He joined the University of Oxford in 2013, where he is currently an Associate Professor with the Department of Engineering Science and a Tutorial Fellow of Oriel College.

$\mathrm{He}$ is the Technical Manager of the EU FP7 project DIWINE. He has authored in excess of 100 papers in leading international journals and conferences, and is a named inventor on over 30 patents. His research interests include communication theory, information theory, and network theory. Dr Coon was a recipient of TRLs Distinguished Research Award for his work on block-spread CDMA, aspects of which have been adopted as mandatory features in the 3GPP LTE Rel-8 standard. He was also a co-recipient of two best paper awards at the ISWCS 2013 and the EuCNC 2014. He received the award for Outstanding Contribution in 2014. He has served as an Editor for the IEEE TRANSACTIONS ON WIRELESS COMMUNICATIONS from 2007 to 2013, the IEEE Transactions ON Vehicular TeChNOLOGy from 2013 to 2016. He has been serving as an Editor for the IEEE WIRELESS COMMUNICATIONS LETTERS since 2016 and the IEEE COMMUNICATIONS LETTERS since 2017. 